\title{
Alternativas al capitalismo. La propuesta de David Schweickart
}

De la oposición de resistencia al análisis de las posibilidades de la sociedad mundial

Si es cierto que, como decía Jean Cocteau, "un buen libro es aquel que siembra abundantemente los signos de interrogación", con Against Capitalism de David Sweickart ${ }^{1}$ estamos a todas luces ante un trabajo de este calibre. En los grandes esfuerzos transformadores, si necesario es el conocimiento de la realidad, no lo es menos la producción de esa sobrerrealidad que permite ver con distancia la realidad inmediata, alzarse sobre ella, fijar las marcas en el camino. Además de un ejercicio necesario para contrarrestar los vértigos del presente, los esbozos como el de este autor nos permiten ingresar en un ámbito especial de la realidad: el de la irrealidad que no es ajeno ni a la fantasía ni a la realidad más real. La construcción de muchas realidades humanas ha de empezar muchas veces por las irrealidades. Como decía el viejo Marx, para realizar la revolución en la tierra necesitamos hacer primero la revolución en el cielo, sin embargo, tenemos que tener claro que no hay una escalera de Jacob, que de las revoluciones en el cielo no se pasa automáticamente a las revoluciones en la tierra. Esta primera revolución es una especie de quijotismo necesario que hay que emprender con coraje para que salten las chispas de las posibilidades. El trabajo de Schweickart es un admirable esfuerzo en este sentido. Este esfuerzo consiste en pasar en virtud de la creación del momento negativo de la oposición de resistencia al análisis de las posibilidades de la sociedad. En este sentido, recoge la experiencia que surge del capitalismo y del socialismo e intenta una nueva lógica económica que no violente a la naturaleza y la humanidad $y$ que sea capaz de enfrentar los problemas mayores de nuestro tiempo, como son la crisis ecológica, las crecientes desigualdades en el mundo y la deshumanización de la vida social.

La obra de Schweickart se inscribe dentro de las corrientes que en el mundo académico intentan restablecer el cordón umbilical que se había roto con los sistemas del mundo físico, para analizar cuál es la gestión que está haciendo la sociedad industrial de los recursos que ha logrado obtener a las alturas de este final de milenio y así estudiar como se puede tratar de reconvertirla. Su análisis parte de la crítica al capitalismo para proponer un socialismo de mercado que ha de llevar a la construcción de una nueva sociedad más igualitaria y humana. Utiliza el marxismo y algunas de sus intuiciones fundamentales para abordar problemas de la sociedad de hoy que no son sólo económicos sino también culturales y sociales.

De la ética de los valores al análisis de las formas de vida

Se trata de una propuesta de economía que no deja fuera la ética. Hoy día está muy de moda hablar de que una ética del futuro exige inaugurar nuevos valores. Sin negar esto, habremos de decir

1. Against Capitalism Westview, Press USA, 1996 (trad. Más allá del capitalismo, Sal Terrae, Barcelona, 1997.) 
que Schweickart se concentra, como es debido, en la búsqueda de una forma de vida que sea realista y viable, que tome en cuenta la responsabilidad hacia el futuro. Como señala Ricoeur: "hasta el momento se considera a alguien como responsable solamente por actos pasados...". Una ética acorde con los tiempos que estamos viviendo tiene que tomar en cuenta la responsabilidad hacia el futuro. "La ética del futuro es fundamentalmente una ética del tiempo que rehabilita el futuro (...). La ética del futuro no es la ética en el futuro, es ética en el presente. Es ética aquí y ahora para que más tarde haya un aquí y un ahora"2. El intento de esbozo de Democracia Económica es movilizador y posibilista a la vez, lo cual es sinceramente de agradecer, pues como dice Ricoeur: "hay que resistirse a la seducción de las esperas puramente utópicas, que no pueden sino exasperar la acción... las esperas deben ser definidas, por lo tanto objetivas y modestas, si se supone deben ser capaces de generar un compromiso responsable", requerimiento que cumple este trabajo. La crisis de la política tanto en oriente como en occidente tiene relación en buena medida con la confusión del futuro (que no está desvinculada de una ruptura con el pasado). "Hay que recordar —dice Jérôme Bindéque la política consiste, primero y ante todo, en estructurar el tiempo"3. "El quehacer propio del hombre político es el porvenir y la responsabilidad frente al porvenir" (Max Weber). El problema de las alternativas surge como problema de cambiar el mundo sin pretender crear algún "otro" mundo. La altemativa senía tratar de ordenar las relaciones económicas.

Las sociedades modemas abundan en especulaciones teóricas, y disponen de una enorme cantidad de información sobre los contextos y los problemas pero, curiosamente, son profundamente ignorantes de la realidad del funcionamiento de sus sistemas prácticos. Lo decisivo no está en los valores que tengamos, sino en las formas de vida. Cualquier reforma que no toque nuestras formas de vida se puede dar como inexistente. Es perentoria una re- forma en nuestros modos de vida ${ }^{5}$, reforma a la que habrán necesariamente de acompañar nuevos valores, pero la reforma habrá que hacerla en el terreno de las prácticas, de lo contrario todo queda en pura prédica, en huero moralismo. Ahora bien, sí hay que decir que a un cambio en las prácticas necesariamente acompañará un cambio de valores. Algunos enfoques proponen cosas tales como una "ética de la acción solidaria"6. Nosotros señalaríamos, apoyando firmemente la propuesta de Schweickart, que en general tenemos que ser más atrevidos en las propuestas y más concretos, puesto que si no, se tiñen de un cierto regusto de "moralina" que no los hace aptos para la digestión de un público cansado de tanta cháchara moral. El libro de Schweickart, en cambio, toca con resolución la ética sin referirse explícitamente a ella. Este es otro punto fuerte.

\section{Los problemas del sistema}

La propuesta de Democracia Económica de David Schweickart pretende enfrentar los problemas cruciales del capitalismo, como el desempleo, la no sostenibilidad y la desigualdad. Aborda con profuso detalle los temas de la eficiencia, del crecimiento económico y de la competitividad, para mostramos que estamos atrapados en una serie de ideas —producción, consumo, trabajo/valor- que identifican riqueza con incremento del saldo monetario, equivocación que se suma a la serie de largos malentendidos que nos están llevando a la perdición.

Respecto a uno de los temas capitales, el del crecimiento, señalemos sólo y desde América Latina que en los años sesenta se empieza a hablar, concretamente en Brasil, Argentina y Chile, de un estancamiento dinámico en los procesos de crecimiento económico. Si en los cincuenta y sesenta se había creído que las tasas de crecimiento creaban empleo adicional y que había una tendencia al pleno empleo, después de una década se comienza a constatar que ésta era una perspectiva muy opti-

2. Citado por Jerôme Bindé, Director de la Unidad de Análisis y Previsión de la UNESCO, La Prensa Gráfica, San Salvador, 8 de noviembre de 1997.

3. Ibid.

4. Hinkelammert, F. Mapa del emperador, Dei, Costa Rica, 1995, p. 258; Cultura de la esperanza y sociedad sin exclusión, Dei, Costa Rica, cap. IV.

5. Ellacuría, I. “Una civilización de la pobreza”, Misterium Liberationis, vol. I.

6. Hinkelammert, F. Mapa del emperador, p. 259. 
mista, que lo que se tendía a producir en realidad es una situación en la que hay dinámica económica, incluso crecimiento, pero que no se integra a toda la población en ella sino que, por el contrario, crece la marginalidad. A esta situación habría que responder con un tipo de desarrollo adecuado ${ }^{7}$ que absorbiera lo que antes eran los sectores marginales y que hoy día se ha convertido en el macrofenómeno de la exclusión ${ }^{8}$. Pero este tipo de desarrollo no sólo no se acaba de hallar, sino que se produce todo lo contrario: desde entonces hasta hoy el tipo de crecimiento económico es cada vez más de estancamiento dinámico. Las tasas de crecimiento siguen siendo positivas (Alemania tiene un 3 por ciento anual, por ejemplo), pero cada año el desempleo aumenta de un 1 a 2 por ciento. Lo

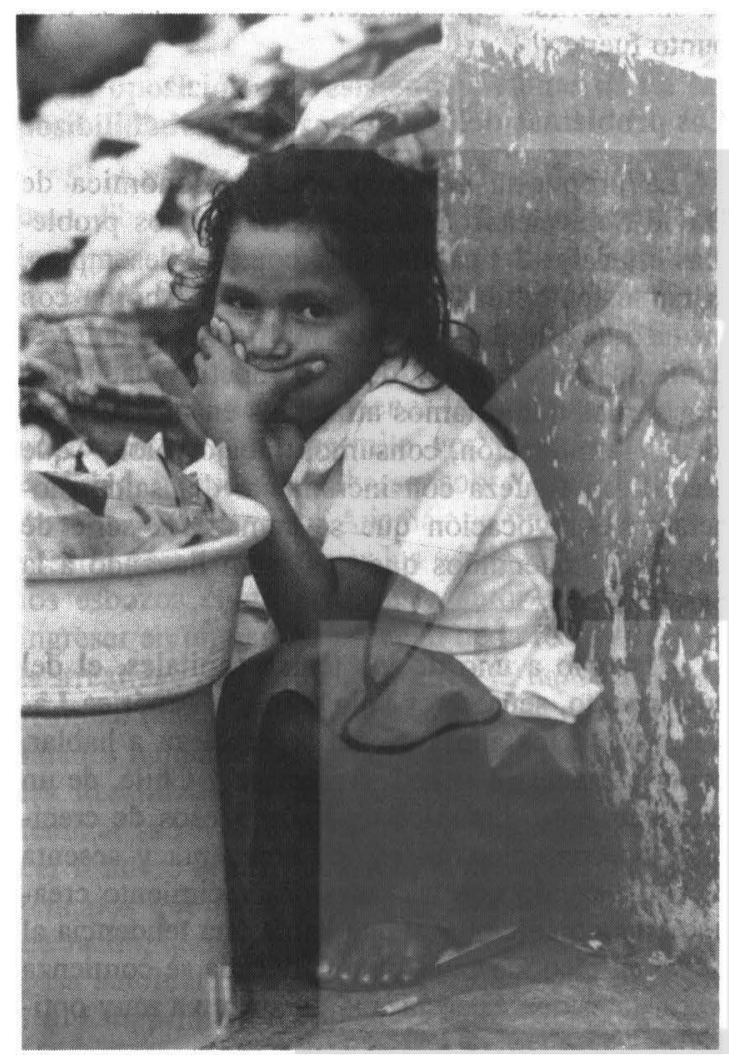

que ocurre es que se ha desvinculado el crecimiento del empleo, se ha producido un encogimiento dinámico: menos asalariados producen beneficios mayores. Como resultado de esto tenemos que la economía en general crece, pero cada vez se benefician sectores más reducidos de la población. Y por encima de todo se ignora el hecho de que el desempleo no es una fatalidad derivada del desarollo tecnológico sino de unas relaciones sociales concretas. El enfoque de la democracia económica pretende buscar el modo de subvertir estas relaciones y muestra que sin estas nuevas relaciones sociales es vano el intento de la "democracia" de presentarse como tal, porque cada vez se pone más de manifiesto que la supuesta igualdad, de la que parte todo proyecto democrático, es una premisa cada día más lejana. No se le escapa que cualquier alternativa a este estado de cosas tiene que poner en jaque a esta dinámica del desempleo" que lleva a la exclusión. La pregunta radical para Schweickart será: ¿qué economía se puede fomentar que sea capaz de superar estas tendencias que causan cada vez resultados más calamitosos? Se trata, como él muestra, de problemas estructurales, por lo tanto, las soluciones también habrán de serlo. Los intentos de superar estas situaciones graves por medio de simples "correcciones" serán totalmente inefectivos, como bien muestra por medio de un concienzudo repaso a las distintas propuestas de los economistas a lo largo de todo este siglo. Los reformismos ensayados hasta la fecha son experiencia acumulada, son dignos de ser tomados en cuenta, pero no son válidos para superar esta tendencia al colapso. Su propuesta de "socialismo de mercado" pretende tener verdaderamente el potencial altemativo para enfrentarlos.

Tiene asimismo potencial para enfrentar el productivismo y replantear la cuestión de la riqueza, tan central para la economía. Curiosamente el grueso de la adquisición de riqueza hoy día aparece no en el campo de los bienes sino en el campo de lo meramente pecuniario. El valor de la producción material, que tanto magnificaron los econo-

7. En esta línea reflexionan muchos autores, citamos solamente a Goulet, Denis. Etica del desarrollo, Estela-lepal, Barcelona, 1965.

8. De modo que la línea divisoria para estar o no integrado en el sistema pasa hoy día por tener o no tener trabajo. Decía un cínico alemán: "¿La felicidad?: ser idiota y tener trabajo". El trabajo y su función en una sociedad alternativa es otro de los ternas colaterales que se abordan con tino en Against Capitalism, pero no tenemos espacio aquí para desarrollarlo.

9. Against capitalism, pp. 103-112. 
mistas clásicos como el marxismo, ha quedado relegado a la periferia del tercer mundo. Los países ricos lo son hoy día porque se dedican al manejo de la información relacionada básicamente con la gestión, la comercialización, el mundo de lo financiero y el marketing. Ese es el universo de lo financiero, el que crece mucho más de prisa que la economía real. Quien maneja esos activos financieros es el que tiene capacidad de compra sobre el mundo. Schweickart se enfrenta a este mito de la teoría económica de llamar "productivas" a toda una serie de actividades que no lo son. Su modelo se enfrenta también a la concepción de que la riqueza es dinero, y su modelo de democracia económica trata de rescatar el valor inmobiliario como uno de los pilares de la economía, lo cual no viene a ser nada nuevo, sino un rescate de cómo era la situación antes de que cayéramos en el absurdo de creer que todo se expresa en dinero. De esta revalorización del valor inmobiliario surge un freno bien poderoso a la tendencia ilimitada a la acumulación. En primer lugar, porque los bienes inmuebles se acumulan con menos facilidad $y$, en segundo lugar, porque también son más concretos: si te apropias de un territorio, una casa, una fábrica, lo haces a costa de otro. Ya no se trata de algo invisible, algo abstracto, como el saldo en una cuenta corriente.

La investigación de Sweickart no elude estos problemas sino que los coloca en el primer plano en que deben estar. Es un hecho que en los últimos años se incrementan los capitales disponibles que no se pueden colocar productivamente, con lo cual crece el capital especulativo. No se trata de que a la clase capitalista le haya dado ahora por el deporte de la especulación, sino que por la dinámica del sistema, ya no hay donde colocar productivamente el capital. La privatización de amplios sectores del estado respondería a la necesidad de colocación de parte del capital especulativo, que en su búsqueda de fuentes de rentabilidad halla en el Estado una oportunidad para colocar estos capitales. Muestra de este crecimiento increíble es el caso de los fondos privados de pensiones. En 1992, la mitad de las acciones de Estados Unidos eran las aseguradoras. Su capital era infinitamente mayor que el de las otras compañías. Cualquier pensamiento sobre las alternativas necesitaba enfocar, además del desempleo que ya hemos apuntado, una estrategia para detener el capital especulativo ${ }^{10}$.

\section{La función del Estado en la sociedad mundial}

Hasta aquí hemos reseñado lo que son, a nuestro juicio, los principales méritos de la propuesta de Democracia Económica de D. Schweickart, pero no podemos dejar de consignar lo que constituye un defecto grave, y es que su marco de referencia es una situación mundial como conglomerado de Estados. Hoy día, si pretendemos ser realistas, tenemos que hablar sin pelos en la lengua de sociedad mundial, como ha mostrado Antonio González". La mundialización es un proceso real. Que haya muchos mitos a su alrededor o que sea fácilmente utilizable ideológicamente no significa que sea, ella misma, un mito o pura ideología. En estos momentos no tomarla en consideración ${ }^{12}$ tiene el peligro de conllevar un nacionalismo que resulta, cuando menos, ingenuo. Como ya advertía un autor hace treinta y dos años: "Asistimos a un recrudecimiento del ultranacionalismo en el mismo momento en que sería necesario descubrir las solidaridades mundiales para enfrentar las dramáticas corrientes que atraviesan el mundo"13.

"Lo hacen, pero no lo saben", decía Marx. "La realidad mundial de nuestro tiempo es el resultado de la interacción de infinidad de acciones políticas, económicas, sociales, mediáticas, militares, culturales,... todas ellas de carácter parcial y dictadas por intereses igualmente parciales. El encuentro - y los desencuentros - entre todas esas acciones produce una realidad global que la gran mayoría sufre, que algunas minorías aprovechan... pero que nadie controla. El mundo marcha en dirección desconocida"14. Intentar ejercer el control, o interrogarnos sobre la posibilidad de controlar

10. Así como también recoger cómo hace Schweickart los de medio ambiente y feminismo, aunque no los consignamos aquí para no exceder los límites de este trabajo.

11. González, Antonio, Un solo mundo, Comillas, Madrid, 1995.

12. El planteamiento de Schweickart no sólo cree que es posible el socialismo de mercado en un solo Estado, sino que no considera en ningún nivel los problemas que plantea la mundialización.

13. Ibld., Goulet, p. 100. Este autor en su Etica del desarrollo propone una solidaridad no restringida.

14. Ortiz, Javier. "El mundo está loco, loco, loco" en Página Abierta, julio, No. 74, Madrid, 1997. 
esferas parciales es intentar ejercer el principio de responsabilidad, por lo que un punto importante en la reflexión sobre las alternativas sería, a nuestro juicio, el de calibrar cuál es efectivamente la función del Estado en la sociedad mundial. Minimizar la importancia de los procesos de mundialización puede resultar fatal a la hora de reunir fuerzas para enfrentar los retos, no sólo porque moralmente estaremos seguramente mal situados ${ }^{15}$, sino también porque desde el punto de vista técnico los esbozos pueden venírsenos abajo. La intuición de Schweickart de que las cooperativas pueden ser una salida económica y éticamente viable al presente régimen de propiedad, puede ser inoperante si se pretende ignorar que se inserta dentro del marco de una realidad que es mundializada ${ }^{16}$, querrámoslo o no.

La empresa mundial, en cambio, sí se sitúa en un escenario mundial. Para las transnacionales el mundo es como un campo de batalla. Este campo está lleno de maraña, por lo tanto hay que limpiarlo; en su jerga, hay que "eliminar las distorsiones del mercado", entendiendo por tales todo lo que impida la libre competencia, todo lo que suba costos. Desde esta perspectiva la empresa transnacional estima que tiene unas necesidades de fluidez que los reglamentos de protección del medio ambiente entorpecen, así como las demandas de educación y salud pública para los trabajadores. Todo esto constituye para las empresas "distorsiones del mercado". La dinámica de la mundialización refuerza el estancamiento dinámico del sistema, puesto que éste se estrangula en la misma medida en que logra eliminar estas llamadas "distorsiones", que no son más que los frenos que los estados ponen a la política del "vale todo". A partir de estas premisas se enfoca la actual política de transformación del Estado, cuyas medidas son en particular aunque no únicamente- sentidas en América Latina. Para Hinkelammert ${ }^{17}$, el Estado es el único sujeto que puede imponer las estrategias de mundialización. La empresa mundial no se puede dedicar por sí misma a implementar sus estrategias sino que utiliza el estado como vector de éstas. De ello concluye que: "por tanto el desmantelamiento del Estado, del cual se habla tanto, es una reestructuración de éste en función de la promoción del proyecto de globalización e inclusive de subvención financiera de su empuje"18. El Estado, por tanto, sería el vehículo de las políticas de "ajuste estructural" que constituyen uno de los fenómenos mayores de la globalización a nivel económico. Dichas políticas de ajuste estructural se apoyan en tres medidas básicas: (1) "Desmantelamiento" del Estado". (2) Apertura del comercio extemo que incluye también los capitales, no solamente las mercancías $^{20}$. (3) Debilitamiento general de los movimientos populares a consecuencia de la limitación de las protecciones legales sobre el trabajo $^{21}$. Como producto de estas medidas se constata el incremento del terrorismo de Estado, con lo cual no se podría afirmar sin más que ha habido un desmantelamiento de esta institución a raíz del proceso de mundialización según Hinkelammert, sino que el estado es más fuerte que antes ${ }^{22}$. Ahora bien, sí le reconoce a éste la pérdida de gran parte del ordenamiento de la economía nacional. Los Esta-

15. "Las naciones continuan restringiendo el principio de la solidaridad y aplicándolo sólo dentro de sus propias fronteras, a pesar de que el desarrollo armonizado sólo pueda ser fruto de una solidaridad universal". Esta solidaridad restringida "es ciega ante las verdaderas exigencias de la comunidad humana e incapaz de superar los bloqueos provocados por el fatalismo interno de esta negación de lo real". Goulet, Denís. Etica del desarrollo, Col. Pensamiento y acción, Barcelona: Ed. Estela-Iepal, 1965.

16. Para exponer muy sucintamente este último punto recurrimos a la exposición que sobre este punto hacía el profesor F. Hinkelammert en lo que constituyó la primera parte del curso "Pensar las alternativas", UCA, San Salvador, octubre, 1997.

17. Y posiblemente también para Schweickart.

18. Goulet, op. cit., p. 17.

19. Que para Hinkelammert no ha resultado ser tal, sino sólo una transformación.

20. Cuestión que hay que estudiar con el máximo detalle, pues ahí podría estribar uno de los hechos mayores de la mundialización.

21. Punto que pondría una vez más sobre el tapete la necesidad de un internacionalismo de nuevo corte.

22. A lo cual cabría responder que "fuerza" y "función policial" no tienen por qué ser necesariamente equiparables. 
dos de este fin de siglo se hallarían en la paradoja de ser los principales impulsores de estrategias que ellos no deciden ${ }^{23}$. El debate interesante sería el de averiguar si es cierto que sin este tipo de estado funcionando como promotor, la estrategia de globalización no funcionaría. De ahí muchos autores concluyen ${ }^{24}$ que la función del Estado está limitándose cada vez más a lo "político" mientras que la economía está en manos de otros, a lo cual habría que responder si no manejamos tal vez una noción obsoleta de política que no nos permite entender correctamente los retos que plantea la mundialización ${ }^{25}$. ¿Se puede sostener hoy día, sin caer en contradicción, que las empresas transnacionales son instituciones puramente económicas y no políticas? Posiblemente tal distinción metodológica sea hoy día menos pertinente que nunca; en un momento en que se constata cómo, por ejemplo, una amenaza de huelga de inversiones es suficiente para hundir a amplios sectores de la población en una crisis. En todo caso, se echan de menos algunas consideraciones sobre política y mundialización en Against Capitalism, defecto que tal vez quede enmendado en la obra sobre la "transición" que está preparando este autor, y que seguramente habrá de iluminar muchos caminos nuevos.

En el terreno económico, como señalábamos, el mundo es como un campo de batalla entre dinosaurios ${ }^{26}$ quienes, con base en la competencia, eficiencia y productividad "marchan adelante y celebran todavía su propia muerte como un logro de su eficiencia". El sistema es como un hombre que corta la rama sobre la cual está sentado y se enor- gullece de la velocidad con la cual logra su fin. Schweickart declaraba en un coloquio ${ }^{27}$ "the nation state si by no means obsolete: it is the only institution capable of contending the force of global capital". Hay que ver varias cosas. En primer lugar, qué función desempeña realmente el Estado en este fin de siglo que obviamente no es el papel que jugaba a comienzos, $y$ esto independientemente de si se juzga obsoleto o no. No se trata tanto de que el Estado sea o no obsoleto, sino de que lo que es definitivamente obsoleta es la concepción política de los estados como sujetos o como actores en este proceso de mundialización. Otra cosa sería ver la fuerza que tienen estos estados para frenar el capital, para imponer condiciones, para hacer transformaciones, o simplemente para gobemar en una coyuntura en que se hace cada vez más dramático el contraste entre el consumo desenfrenado de una parte de la población y el hambre de otros. ¿Hasta qué punto va a aguantar el sistema las contradicciones entre gigantes y enanos? Queda patente una vez más que "la revolución no debe verse ya como la locomotora que jala el tren de la humanidad en una línea directa hacia una nueva fase superior, sino como el freno de seguridad del tren que puede llevar al abismo si no es detenido"23. Si, como decía Wittgenstein, "una nueva palabra es como una semilla fresca que se arroja al terreno de la discusión"29. Democracia Económica es una de ellas y, por cierto, bien fecunda en sugerencias.

Judit Ribas San Salvador, 28 noviembre 1997.

23. El Fondo Monetario Internacional e instancias parejas se apoyan prácticamente en el Estado.

24. Entre ellos Hinkelammert.

25. Cfr., Ribas, Judit. "Poder y política en el contexto de la mundialización”, Seminario Zubiri-Ellacuría, Mundialización y Liberación, Managua, 1995.

26. Por tomar una metáfora de Hinkelammert. Con palabras de José del Val (mexicano, mestizo), Director del Instituto Indigenista de la OEA: "El mundo, con la globalización, se concibe como un mercado y, por tanto, sus agentes son las empresas transnacionales que sólo tienen que rendir cuentas a sus accionistas. No hay lógica de Estado, no hay lógica de desarrollo, de población", Tegucigalpa, Honduras, I Cumbre Indígena de Centroamérica. El problema es que el Estado ha dejado de ser soberano. El pueblo, si algún día lo fue, dejó de serlo mucho antes.

27. Curso de doctorado, UCA, San Salvador, octubre, 1997.

28. Femández Buey, F. y Muntaner, Charles. "Marxismos contra corriente: sopesando la década de los ochenta", en Realidad, No. 48, San Salvador, noviembre-diciembre, 1995, p. 1106.

29. Wittgenswtein, Ludwig. Observaciones, México: Ed. Siglo XXI, 1981, p. 14. 